\title{
Comparison of the Multi-Band Hubbard and Heisenberg Models on Small Finite Clusters
}

\author{
J. MATYSIAK* AND R. LEMAŃSKI \\ Institute of Low Temperature and Structure Research, Polish Academy of Science, \\ Okólna 2, 50-422 Wrocław, Poland
}

\begin{abstract}
We perform exact numeric calculations for a few versions of the two-orbital Hubbard model on the four site cluster. We show that the conventional spin 1 Heisenberg Hamiltonian can be obtained in the strong coupling limit, but only for some versions of the two-orbital Hubbard model. Then we propose a modified version of the Heisenberg Hamiltonian, that we call the two-orbital Heisenberg model, which is relevant in those cases, where the conventional spin 1 Heisenberg model fails.
\end{abstract}

DOI: 10.12693/APhysPolA.133.405

PACS/topics: 31.15.vq, 75.10.Jm, 75.10.Pq

\section{Introduction}

It is well known that the spin $1 / 2$ Heisenberg Hamiltonian can be derived from the single-band Hubbard Hamiltonian at half filling in the large interaction limit [1-6]. However, as far as we know, not much consideration has been given to studies of relationships between multibands Hubbard models and the Heisenberg model with spins higher than $1 / 2$. This question is relevant for correlated materials with orbital degeneracy, i.e. for most of the transition-metal compounds. In particular, it is important in the theoretical analysis of single molecular magnets (SMMs). Magnetic molecules are usually described by phenomenological spin model Hamiltonians, which contain the Heisenberg term as a dominant one (e.g. $[7,8])$. There are also attempts to describe these systems from the first principles using the DFT method and by applying the Falicov-Kimball model [9]. But recently, it has been proposed to describe SMMs using the multi-band Hubbard model (HM) in the large interaction limit combined with DFT calculations [10]. Here we examine this approach by performing exact numerical calculations for the four-site ring with two orbitals per site, so we deal with the two-band HM. Such a small ring size was chosen to allow for performing the exact numeric calculations. Since we deal with small clusters, henceforth we replace the name 'multi-band HM' by 'multiorbital HM'.

In this paper, we study two versions of the two-orbital HM: the standard HM represented by the Hamiltonian $H_{H M}$, that contains only the direct Coulomb-type interactions, and the developed HM (DHM) represented by the Hamiltonian $H_{D H M}$, that includes in addition the exchange interactions $\left(H_{H M}\right.$ was already considered in [11-13] and $H_{D H M}$ in $[14,15]$ but for other systems). In fact, we consider two types of $H_{D H M}$ assuming two dif-

*corresponding author; e-mail: j.matysiak@int.pan.wroc.pl ferent possible cases for the hopping amplitudes of electrons. The Hamiltonians $H_{H M}$ and $H_{D H M}$ are as follows:

$$
\begin{gathered}
H_{H M}=\sum_{i \neq j, m, m^{\prime}, \sigma} t^{m m^{\prime}} c_{i m \sigma}^{\dagger} c_{j m^{\prime} \sigma}+U \sum_{i m} n_{i m \downarrow} n_{i m \uparrow} \\
\quad+\frac{1}{2} \sum_{i, m \neq m^{\prime}, \sigma}\left[U^{\prime} n_{i m \sigma} n_{i m^{\prime} \bar{\sigma}}+U^{\prime \prime} n_{i m \sigma} n_{i m^{\prime} \sigma}\right] \\
H_{D H M}=H_{H M} \\
+\frac{1}{2} \sum_{i, m \neq m^{\prime}, \sigma}\left[J c_{i m \sigma}^{\dagger} c_{i m^{\prime} \bar{\sigma}}^{\dagger} c_{i m \bar{\sigma}} c_{i m^{\prime} \sigma}+J c_{i m \sigma}^{\dagger} c_{i m \bar{\sigma}}^{\dagger} c_{i m^{\prime} \bar{\sigma}} c_{i m^{\prime} \sigma}\right]
\end{gathered}
$$

where $i$ and $j$ denote nearest-neighbour sites, $m, m^{\prime}$ label orbitals and $\sigma, \bar{\sigma}$ label spins of electrons $(\bar{\sigma}=-\sigma)$. $U, U^{\prime}$ and $U^{\prime \prime}$ describe the Coulomb type on-site interactions between two electrons: $U$ - on the same orbital and $U^{\prime}\left(U^{\prime \prime}\right)$ - on different orbitals with opposite (parrallel) spins, respectively. $J$ represents the on-site exchange coupling, but it also enters the interaction constants in $H_{H M}$. Indeed, we take $U^{\prime}=U-2 J, U^{\prime \prime}=U-3 J$, as it was derived in [12]. So the Hamiltonian $H_{H M}$ is not equivalent to $H_{D H M}$ with $J=0$.

Our aim is to compare the lowest parts of energy spectra of $H_{H M}$ and $H_{D H M}$ with the spectra of effective spin models derived from the perturbation theory. Since for all these models, subspaces with various total spin $\mathrm{S}$ are orthogonal to each other, we simplify presentation of our results by considering only states with the total spin $S=0$. However, other subspaces should of course be included if one would want to compare calculated results with experimental ones.

\section{The lowest parts of energy spectra}

Contrary to the single orbital model, in the multi orbital case, the states with different spin configurations have, in general, different energies even in the limit where $t^{m m^{\prime}}=0$ for all hopping constants. Then, in order to obtain the effective Hamiltonian with only spin degrees of freedom, we need to restrict our considerations to a 
subspace with the lowest energy, where all unperturbed states are degenerate.

The number of all states of the cluster with the total spin $S=0$ is 4900 . When all $t^{m m^{\prime}}=0$, then energies of these states assume 26 different values both in the case of $H_{H M}$ and $H_{D H M}$, but they are not the same in the two cases. It also turns out that the degenerations of these states are different in the both cases. Indeed, the ground state degeneracy amounts 6 for $H_{H M}$ and 19 for $H_{D H M}$.

Figure 1 presents the lowest parts of energy spectra of the two orbital HM in the limit where all hopping constants $t^{m m^{\prime}}=0$ (i.e. $t^{11}=t^{22}=t^{12}=t^{21}=0$ ), obtained for the four site chain with two orbitals on each site. The parameters $U=6 \mathrm{eV}, J=0.3 \mathrm{eV}$ are chosen in accordance with the calculations reported in [10].

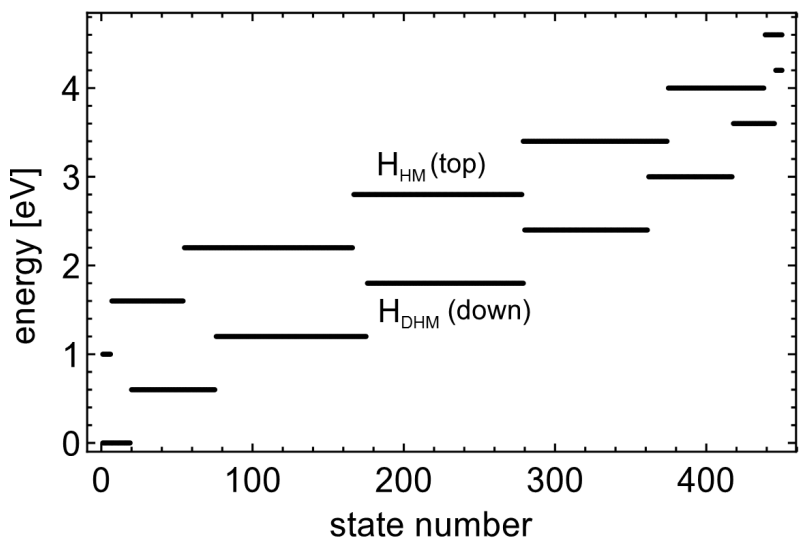

Fig. 1. Comparison of the lowest parts of energy spectra of $H_{H M}$ and $H_{D H M}$ for the subspace with the total spin $S=0$ obtained in the limit where all hopping constants $t^{m m^{\prime}}=0, U=6 \mathrm{eV}$ and $J=0.3 \mathrm{eV}$. To enable the comparison the spectra are separated (here the spectrum of $H_{H M}$ is moved up by $1 \mathrm{eV}$ ).

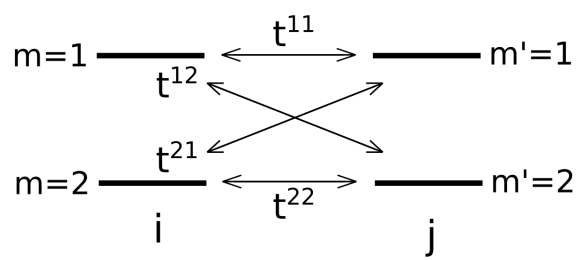

Fig. 2. Labelling of the hopping amplitudes $t^{m m^{\prime}}$ between the sites $i$ and $j$ in the two orbital HM. $m, m^{\prime}$ are orbital indices.

In the two orbital HM there are, in general, four independent hopping amplitudes $t^{m m^{\prime}}$, which are illustrated in Fig. 2. Here we restrict our studies only to the two special cases: a) $t^{11}=t^{22}=t, t^{21}=t^{12}=0$ and b) $t^{11}=t^{22}=t^{21}=t^{12}=t$. For the finite hopping amplitudes $t^{m m^{\prime}}$, we observed a slight splitting of each degenerate subset of eigenvalues presented in Fig. 1. Obviously, the splittings in the cases a) and b) are different.
Now we would like to focus our attention on the DHM. Our task is to compare the lowest part of energy spectrum of $H_{D H M}$ with the spectrum of the spin-1 Heisenberg Hamiltonian $H_{H \text { eis }}^{S=1}$ applied to the ring of four spins $S_{i}=$ 1

$$
H_{\text {Heis }}^{S=1}=\Gamma \sum_{i j} \mathbf{S}_{\mathbf{i}} \mathbf{S}_{\mathbf{j}}
$$

and the lowest part of the spectrum of the multi-orbital Heisenberg Hamiltonian $H_{\text {Heis }}^{\text {morb }}$

$$
H_{\text {Heis }}^{\text {morb }}=\sum_{m m^{\prime} i j} \Gamma_{m m^{\prime}} s_{i m} s_{j m^{\prime}},
$$

where $\mathbf{S}_{i}=\left(S_{i}^{x}, S_{i}^{y}, S_{i}^{z}\right)$ refers to the ion's spin 1 at the site $i$ and $s_{i m}$ refers to the electron's spin $1 / 2$ on a single orbital $m$. We point out here that the dimensions of Hilbert spaces of admitted states of the Hamiltonian (4) and (3) are different. Indeed, in our system the number of all admissible states amounts 19 in the case of $H_{\text {Heis }}^{S=1}$ and 70 in the case of $H_{\text {Heis }}^{\text {morb }}$. However, the Hamiltonian (4) is acting only within the subspace of the lowest energy eigenstates of $H_{D H M}$ (they are degenerated in the atomic limit) that also contains 19 states.

Since in our calculations we put $U=6 \mathrm{eV}, J=0.3 \mathrm{eV}$, $t^{m m}=0.1 \mathrm{eV}$, then from the perturbation theory for $H_{\text {Heis }}^{\text {morb }}$ we get the exchange parameters being approximatively equal to $\Gamma_{m m^{\prime}}=\frac{2\left(t^{m m^{\prime}}\right)^{2}}{U+J}=0.00318 \mathrm{eV}$.

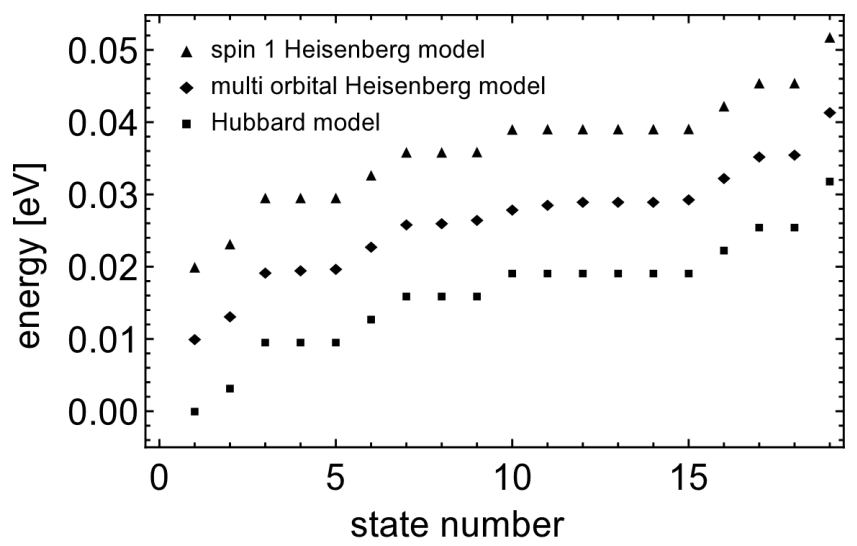

Fig. 3. Comparison of the lowest parts of energy spectra of the models $H_{D H M}, H_{H e i s}^{S=1}$ and $H_{\text {Heis }}^{m \text { orb }}$ for $t^{m m}=$ $0.1 \mathrm{eV}, t^{m m^{\prime}}=0$ for $m \neq m^{\prime}, U=6 \mathrm{eV}, J=0.3 \mathrm{eV}$ and $\Gamma=0.00318 \mathrm{eV}$. To enable the comparison the spectra are separated from each other by $0.01 \mathrm{eV}$.

The result of the comparison of the energy spectra is illustrated in Figs. 3 and 4. Figure 3 shows the data for the case a) and Fig. 4 for the case b). In both figures we compare the lowest 19 eigenvalues of the models: $H_{D H M}$, $H_{\text {Heis }}^{S=1}$ and $H_{\text {Heis }}^{\text {morb }}$. Based on this comparison we admit that the Hamiltonian $H_{H e i s}^{S=1}$ may, indeed, play a role of the effective Hamiltonian when $t^{11}, t^{22} \gg t^{12}, t^{21}$, but it is not the case in the region where $t^{11}, t^{22} \sim t^{12}, t^{21}$. On the other hand, the Hamiltonian $H_{\text {Heis }}^{\text {morb }}$ seems to be more universal, as it is relevant in the both cases. 


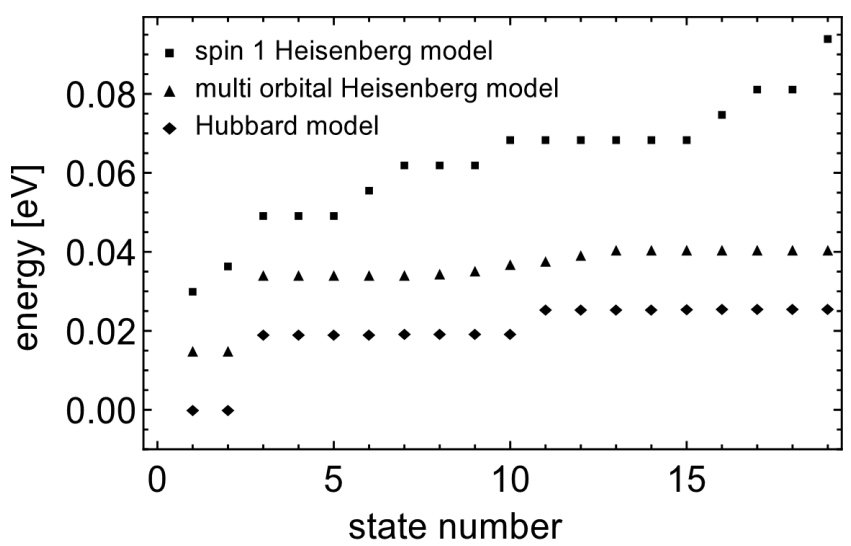

Fig. 4. Comparison of the lowest parts of energy spectra of the models $H_{D H M}, H_{H e i s}^{S=1}$ and $H_{\text {Heis }}^{\text {morb }}$ for $t^{m m^{\prime}}=$ $0.1 \mathrm{eV}$ for all $m, m^{\prime}, U=6 \mathrm{eV}, J=0.3 \mathrm{eV}$ and $\Gamma=0.00318 \mathrm{eV}$. To enable the comparison the spectra are separated from each other by $0.015 \mathrm{eV}$.

We also performed calculations for the Hamiltonian $H_{H M}$, where the exchange interaction terms are omitted $[11,12]$. It's overall spectrum is similar to the one obtained for $H_{\text {Heis }}^{S=1}$, but their lower parts differ considerably. In particular, the lowest energy subspace now contains only 6 states, as opposed to 19 in the previous case (for the total spin of the whole molecule $S=0$ ).

Then, the result of comparison of energy spectra of the Hamiltonians $H_{H M}, H_{H e i s}^{S=1}$ and $H_{\text {Heis }}^{\text {morb }}$ applied to those the lowest 6 energy levels is illustrated in Fig. 5.

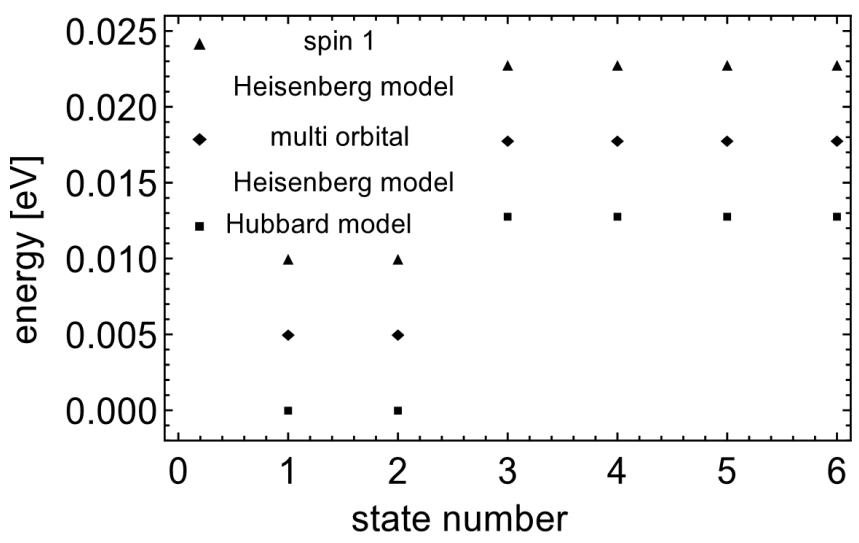

Fig. 5. Comparison of the lowest parts of energy spectra of $H_{H M}, H_{\text {Heis }}^{S=1}$ and $H_{\text {Heis }}^{\text {morb }}$ for $t^{m m}=0.1 \mathrm{eV}$, $t^{m m^{\prime}}=0$ for $m \neq m^{\prime}, U=6 \mathrm{eV}$ and $J=0.3 \mathrm{eV}$. To enable the comparison the spectra are separated from each other by $0.005 \mathrm{eV}$.

\section{Summary and conclusions}

We performed exact numeric calculations for the four site ring, with two orbitals and two electrons per site, using the two-orbital Hubbard Hamiltonians $H_{H M}$ and $H_{D H M}$. We have found that the energy spectra of the spin 1 Heisenberg model $H_{H \text { eis }}^{S=1}$ and the lowest part of the spectra of $H_{D H M}$ are equivalent only when the hopping amplitudes between orbitals of the same type are much larger than between different ones. On the other hand, the multi-orbital Heisenberg model $H_{\text {Heis }}^{\text {morb }}$ reproduces satisfactory the lowest part of energy spectrum of $H_{D H M}$ both for the case a) $t^{11}=t^{22}=t, t^{21}=t^{12}=0$ and b) $t^{11}=t^{22}=t^{21}=t^{12}=t$.

We also found that the lowest part of energy spectrum of the simplified multi-orbital Hubbard Hamiltonian $H_{H M}$ (without the exchange terms) can also be approximately reproduced by $H_{H e i s}^{\text {morb }}$.

So we suggest to use the model $H_{\text {Heis }}^{\text {morb }}$ in further studies of SMM's materials, as it seems to represent more adequately a wider class of microscopic models of correlated electrons in the large interaction limit than the spin $S$ Heisenberg model $H_{\text {Heis }}^{S}$.

\section{References}

[1] A.H. MacDonald, S.M. Girvin, D. Yoshioka, Phys. Rev. B 37, 9753 (1988).

[2] C.L. Cleveland, R. Medina, Am. J. Phys. 44, 44 (1976).

[3] K.A. Chao, J. Spałek, A.M. Oleś, J. Phys. C 10, L271 (1977).

[4] K. A. Chao, J. Spałek, A. M. Oleś, Phys. Rev. B 18, 3453 (1978).

[5] R. Micnas, J. Ranninger, S. Robaszkiewicz, Rev. Mod. Phys. 62, 113 (1991).

[6] E. Manousakis, Rev. Mod. Phys. 63, 1 (1991).

[7] M. Affronte et al., Phys. Rev. B. 68, 1 (2003).

[8] P. Kozłowski, G. Kamieniarz, J. Nanosci. Nanotechnol. 11, 9175, (2011).

[9] B. Brzostowski, R. Lemański, T. Ślusarski, D. Tomecka, G. Kamieniarz, J. Nanopart. Res. 15, 1528 (2013).

[10] A. Chiesa, S. Carretta, P. Santini, G. Amoretti, E. Pavarini, Phys. Rev. Lett. 110, 15 (2013).

[11] C. Lacroix-Lyon-Caen, M. Cyrot, Solid State Commun. 21, 837 (1977).

[12] R. Frésard, G. Kotliar, Phys. Rev. B. 56, 12909 (1997).

[13] J. Kim, M. Y. Choi, G. S. Jeon, Phys. Rev. B 92, 125127 (2015).

[14] C. Castellani, C.R. Natoli, J. Ranninger, Phys. Rev. B 18, 4945 (1978).

[15] J.M. Pizarro, M.J. Calderón, J. Liu, M.C. Muñoz, E. Bascones, Phys. Rev. B 95, 075115 (2017). 